\section{Elimination of Internal Restriction Enzyme Sites from a Bacterial Lumi- nescence (luxCDABE) Operon}

BioTechniques 24:56-58 (January 1998)

The $\operatorname{lu} x A$ and $\operatorname{lu} x B$ genes of luminescent bacteria, most frequently from Vibrio spp., are widely used as reporter genes $(3,10)$. The heterodimer $(\alpha \beta)$ luciferase enzyme catalyzes oxidation of the reduced flavin mononucleotide $\left(\mathrm{FMNH}_{2}\right)$ and a long-chain fatty aldehyde, resulting in emission of light. In vitro, dodecanal or nonanal are used as preferred exogenous aldehyde substrates for bacterial luciferases (3).

In addition to the $\operatorname{lux} A B$ genes, the lux structural operons of luminescent bacteria also direct the synthesis of a fatty acid reductase complex (encoded by the luxCDE genes), which synthesizes tetradecanal, a natural substrate of luciferase (7). Introduction of the lux$C D A B E$ operon of a luminescent insect pathogen bacteria, Xenorhabdus luminescens, into Escherichia coli resulted in a luminescent phenotype $(5,6)$ because of the expression of the luciferase enzyme and synthesis of the tetradecanal substrate. This indicates that luminescence of these recombinant $E$. coli cells is independent of the exogenous substrate. In this regard, the lux$C D A B E$ operon is a more easily applicable system than the $\operatorname{lux} A B$ genes, in which addition of an exogenous substrate is required for light emission. A particular advantage of using the lux operon of $X$. luminescens as a reporter system is that the LuxAB protein of this species is the most thermostable of all the known bacterial luciferases (3).

A factor that may limit the use of the luxCDABE operon of $X$. luminescens in genetic engineering is the presence of several internal restriction enzyme sites. The operon can be isolated as a 6960-bp EcoRI fragment $(5,6)$, and while it has no SacI, KpnI, SmaI, XmaI, BamHI, SalI and PstI sites, there are HindIII, SphI and XbaI sites in the operon (Figure 1). Because these sites are common in the multiple cloning sites (MCSs) of many plasmid vectors, we aimed to eliminate them from the lux operon of X. luminescens to facilitate manipulation of the operon. The sites were eliminated by site-directed mutagenesis, and we produced a lux into the symmetrical MCS of plasmid pMTL25 (2).

Site-directed mutagenesis was performed on plasmid pLITE27 containing the 6960-bp EcoRI lux fragment of $X$. luminescens in pUC118 (6). The seven mutagenic primers used for the elimination of the restriction sites from the lux operon (Figure 1) were designed in such a way that the base-pair exchanges do not alter the encoded amino acids of the Lux proteins. An additional primer was used to eliminate an external HindIII site flanking the lux operon in pLITE27. The elimination of an external restriction site is required for removal of the wild-type plasmid molecules during the mutagenesis process (4). The sequence of the external primer we used is: $5^{\prime}$-GGCCAGTGCCAACCTTGCATGC-3', where the mutant restriction site is underlined with the exchanged nucleotide in boldface. The mutagenesis was performed according to the method described by Deng and Nickoloff (4) with two modifications. First, T4 DNA polymerase was replaced by T7 DNA polymerase (New England Biolabs, Beverly, MA, USA) in the second-strand synthesis reaction. Like T4 DNA polymerase, T7 DNA polymerase does not perform strand displacement synthesis (an important requirement in site-directed mutagenesis; Reference 1). The polymerization by T7 DNA polymerase, however, is faster and more efficient cassette by cloning the mutant operon

than that by T4 DNA polymerase (1). This is an advantage if the template molecule is relatively large, as is the case with pLITE27 (about $10 \mathrm{~kb}$ ). Second, we incorporated all eight primers into a single second-strand synthesis reaction. Previously, Ray et al. reported achieving eleven point mutations in four rounds of site-directed mutagenesis (8).

Following the second transformation of the mutagenesis procedure, about 200 primary transformants grown on LB/ampicillin agar plates were screened for luminescence. The plates were taken into a darkroom, and after allowing 2-3 min for the eyes to accommodate to the dark, 14 highly luminescent colonies were identified by visual examination of the plates with the naked eye. Plasmid DNA was isolated from these colonies using the alkaline lysis miniprep method (9). A HindIII diagnostic digest revealed that eight of the isolated plasmids are not cut by this enzyme. Of these eight plasmids, four that were purified from colonies displaying the same level of luminescence as the original pLITE27/ DH5 $\alpha$ strain (DH5 $\alpha^{\mathrm{TM}}$ from Life Technologies, Gaithersburg, MD, USA) (data not shown) were selected for further examination. Each of them was digested with EcoRI, and the 6960-bp lux fragments were gel-purified using the GLASSMAX ${ }^{\circledR}$ DNA Isolation Spin Cartridge System (Life Technologies). The isolated fragments were treated with $S p h I, X b a I$ and, once more, HindIII restriction enzymes. Three fragments, obtained from plasmids designated pLITE2, pLITE5 and pLITE7, were not cut by either enzyme, indicating that all

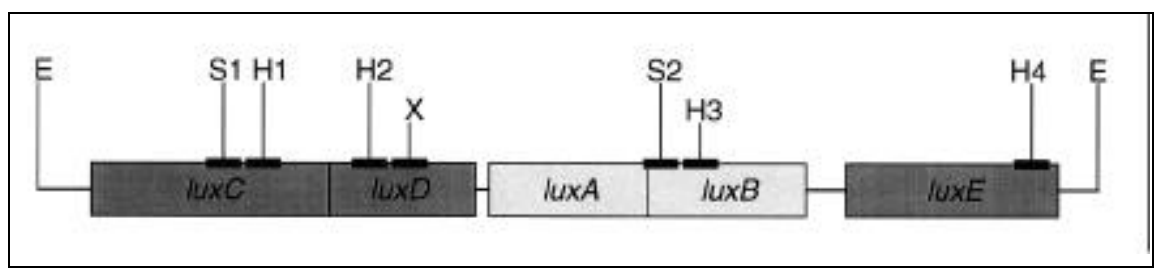

Figure 1. Physical map of the lux operon of $X$. luminescens. Only the restriction sites used for cloning (E: EcoRI) and to be eliminated (H: HindIII; S: SphI; X:XbaI) are shown. In the vicinity of the restriction sites, the positions of the mutagenic primers are labeled by short black lines. The following primers were used: S1: 5'-CGGTAGAGCACGCGCCATCTT-3'; H1: 5'-AAAAGAAAGCCTGTTTGCTGG-3'; H2: 5'-TGTTGGCTTCCAGCTTATCTG-3'; X: 5'-CGGATAATCTGGATTTTGAAG-3'; S2: 5'-GTATAGTTCGTATGCAGGAAA-3'; H3: 5'-GCGGAGGAAGCGTGCTTATTG-3'; and H4: 5'-GGTGTGCTTTGAGCTTAACCG- $3^{\prime}$. In the primer sequences, the mutant restriction sites are underlined with the exchanged nucleotide in bold-face. Phosphorylated primers were synthetized by Oligos Etc. (Wilsonville, OR, USA). The figure is not drawn to scale. 
of the seven target restriction sites had been eliminated from the luxCDABE operon. A representative digestion of the mutant lux fragment of pLITE2 compared to the wild-type fragment of the original plasmid, pLITE27, is shown in Figure 2.

The mutant lux fragment of plasmid pLITE2 was then cloned into the single EcoRI site of plasmid pMTL25, which has several symmetrical restriction sites flanking the single EcoRI site (2). The cloning resulted in the lux operon cassette plasmid pLITE201, from which the luxCDABE operon of $X$. luminescens can be recovered as a single fragment by BamHI, EcoRI, HindIII, KpnI, PstI, SalI, SmaI, SphI and XbaI, or by double digestion using either

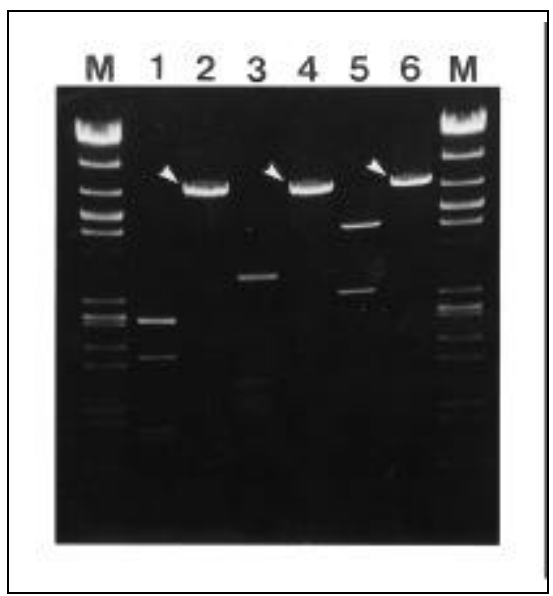

Figure 2. Restriction digestion of the isolated wild-type and mutant lux operon DNAs. Lanes 1, 3 and 5: wild-type; lanes 2, 4 and 6: mutant EcoRI lux DNA fragments digested by HindIII (lanes 1 and 2), SphI (lanes 3 and 4) and $X b a I$ (lanes 5 and 6) and separated on a $1 \%$ agarose gel in TAE buffer (10) in the presence of ethidium bromide. The 6960-bp mutant lux fragments resistant to the restriction enzymes are labeled by arrowheads. Note that digestion of the isolated, linear, wild-type lux fragment with enzymes HindIII and SphI should result in five and three restriction fragments, respectively (Figure 1). The presence of only four and two bands in lanes 1 and 3, respectively, is due to the very similar size of two fragments generated in both digestions, resulting in double bands indicated by the relatively stronger fluorescence of the largest fragments in lanes 1 and 3. Lanes $M$ are molecular weight markers (a mixture of $\lambda$ DNA digested by HindIII and HindIII $+E c o$ RI). Fragment sizes from the top to bottom are: $23.13+21.22$ (c), $9.42,6.56,5.15+4.97$ (c), $4.36+4.28$ (c), 3.53 , $2.32,2.03+2.03(\mathrm{c}), 1.90,1.58,1.33,0.98,0.83$, 0.56 (in kb). Some bands in the marker lanes [indicated by "(c)"] are co-migrating and cannot be separated under the applied conditions of the electrophoresis.
HindIII, PstI or SphI plus either NcoI, StuI or XhoI (data not shown). Note that sequencing of the entire mutant lux operon of pLITE201 has not been performed, and therefore putative mutations, which however do not influence either the restriction pattern or the biological activity of the operon, might occur because of the fidelity of T7 polymerase.

In conclusion, we have constructed a lux $C D A B E$ operon, having no internal restriction sites for enzymes that are most common in different cloning vectors. This operon could be used for many studies, e.g., as a reporter for gene expression, for tracking bacterial populations and for biosensors $(3,7,10)$. Because addition of an exogenous luciferase substrate is not a requirement for light emission using the lux $C D A B E$ operon, this construction might be very valuable in situations where addition of the substrate is difficult, e.g., in environmental studies. In our laboratory, we are currently using the $\operatorname{lux} C D A B E$ operon of $X$. luminescens as a reporter of cellular viability of immobilized bacterial cells in fermentation studies.

\section{REFERENCES}

1.Bebenek, K. and T.A. Kunkel. 1989. The use of native T7 DNA polymerase for site-directed mutagenesis. Nucleic Acids Res. 17:5408.

2.Chambers, S.P., S.E. Prior, D.A. Barstow and N.P. Minton. 1988. The pMTL nic cloning vectors. I. Improved pUC polylinker regions facilitate the use of sonicated DNA for nucleotide sequencing. Gene 68:139-149.

3.Chatterjee, J. and E.A. Meighen. 1995. Biotechnological applications of bacterial bioluminescence (lux) genes. Photochem. Photobiol. 62:641-650.

4.Deng, W.P. and J.A. Nickoloff. 1992. Site-directed mutagenesis of virtually any plasmid by eliminating a unique site. Anal. Biochem. 200:81-88.

5.Frackman, S., M. Anhalt and K.H. Nealson. 1990. Cloning, organization, and expression of the bioluminescence genes of Xenorhabdus luminescens. J. Bacteriol. 172:5767-5773.

6.Marincs, F. and D.W.R. White. 1994. Immobilization of Escherichia coli expressing the lux genes of Xenorhabdus luminescens. Appl. Environ. Microbiol. 60:3862-3863.

7.Meighen, E.A. 1993. Bacterial bioluminescence: organization, regulation, and application of the lux genes. FASEB J. 7:1016-1022.

8.Ray, F.A., E.M. Miller and J.A. Nickoloff. 1995. Efficient marker rescue and domain replacement without fragment subcloning. Anal. Biochem. 224:440-443.

9.Sambrook, J., E.F. Fritsch and T. Maniatis. 1989. Molecular Cloning: A Laboratory Man- ual, 2nd ed. CSH Laboratory Press, Cold Spring Harbor, NY.

10.Stewart, G.S.A.B. and P. Williams. 1992 lux genes and the applications of bacterial bioluminescence. J. Gen. Microbiol. 138:12891300 .

This work was supported by the New Zealand Foundation for Research Science and Technology. Address correspondence to Dr. Ferenc Marincs, AgResearch, Grasslands Research Centre, Tennent Drive, Private Bag 11008, Palmerston North, New Zealand. Internet: marincsf@agresearch. cri.nz

Received 13 May 1997; accepted 22 July 1997.

\section{Christine R. Voisey and} Ferenc Marincs AgResearch, Grasslands Research Center Palmerston North, New Zealand 